\title{
Dynamic analysis of flexible-macro rigid-micro manipulator
}

\author{
Zhang Piaoshi, Liu Jubao, Li Guowei, Gao Sheng
}

School of Mechanical Science and Engineering, Northeast Petroleum university, Heilongjiang

Daqing 163318, China

Keywords: flexible-macro rigid-micro; manipulator; dynamic modeling; characteristic analysis

\begin{abstract}
Flexible-macro rigid-micro manipulator is a special structure robot system, which is suitable to perform large motion with precise operation task. For a planar manipulator with two flexible-macro manipulators and two rigid-micro manipulators, this paper performs dynamic analysis of flexible-macro rigid-micro manipulator. Detailed simulation results are given to gain the relationship between flexible-macro rigid-micro and its parameters. The theory basis is provided for studying the structure design and structure parameter optimization of flexible-macro rigid-micro manipulator.
\end{abstract}

\section{Introduction}

With the development of the national economy and the progress of science and technology, flexible manipulators have an important direction of robotics research in recent years. It has a wide range of applications in many engineering fields, such as space equipment, modern manufacturing, the IC packaging equipment, printing industry and MEMS [1].

From the perspective of the research achievements of flexible manipulators in recent years, the assumed modes method, Finite Element Method and lumped mass method can effectively describe the deformation of flexible manipulators [2] [3].

\section{Descriptions of flexible-macro rigid-micro structure manipulator}

The flexible-macro rigid-micro structure manipulator refers to attaching a small manipulator at the end of a large manipulator. The large manipulator takes the base as the reference object, which is called as the macro manipulator [4]. The small manipulator takes large manipulator as the reference object and has full freedom, which is called as the micro manipulator [5]. From a structural point of view, the macro manipulator can use lightweight materials under the premise that the rigidity of the micro manipulator should be ensured, so the flexible-macro rigid-micro manipulator has the property of universality.

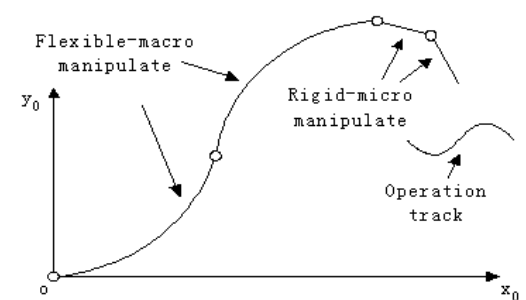

Fig.1. The plane manipulate model

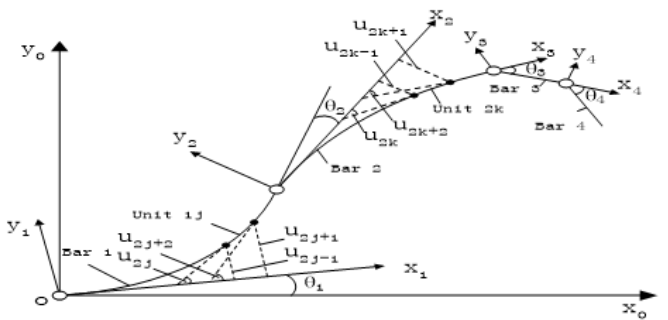

Fig.2. The plane manipulate parameters description

Fig. 1 shows a planar 2F2R macro-micro manipulator. $2 \mathrm{~F}$ means that the flexible-macro manipulator has two degrees of freedom which is used to locate the wide range work space. $2 \mathrm{R}$ means that the rigid-micro manipulator has two degrees of freedom which is used to compensate the error caused by the deformation of the flexible-macro manipulator.

Fig.2 shows the 2F2R macro-micro manipulator system model, in which there are local coordinate system, joint angle variable and the definition of local deformation of flexible bar. There are $\mathrm{m}$ bars which are defined by the connecting conditions of rotary joint, consist of a rigid bars and $b$ flexible bars. For the flexible bar $i$, discrete it into $n_{i}$ units, length of each unit is $l_{i}$, any unit of bars is represented by $\mathrm{ij}$, $\mathrm{i}$ is the number of the bar, $\mathrm{j}$ is the jth unit of the bar. According to 
above-mentioned definitions, the second flexible manipulator is divided into $\mathrm{n}_{2}$ units, each of which contains 2 nodal points. For each unit ij, work out the kinetic energy $\mathrm{T}_{\mathrm{ij}}$ and the potential energy $\mathrm{V}_{\mathrm{ij}}$ by use of generalized coordinates and generalized speed which describes the system motion, and then accumulate them to acquire the total kinetic energy and the total potential energy of the system. Substituting it for the Lagrange equation, then obtains system dynamics equation

$$
M(q)\left[\begin{array}{c}
\ddot{\theta}_{M} \\
\ddot{\theta}_{m} \\
\ddot{u}
\end{array}\right]+H(q, \dot{q})\left[\begin{array}{c}
\dot{\theta}_{M} \\
\dot{\theta}_{m} \\
\dot{u}
\end{array}\right]+K\left[\begin{array}{c}
\theta_{M} \\
\theta_{m} \\
u
\end{array}\right]=\left[\begin{array}{c}
\tau_{M} \\
\tau_{m} \\
0
\end{array}\right]
$$

In the formula: $\mathrm{M}$ is the system positive definite inertia matrix; $\mathrm{H}$ is one item that related to the coriolis-exciting-force and the centripetal force; $K$ is the stiffness matrix; $\theta=\left[\begin{array}{ll}\theta_{M} & \theta \mathrm{m}\end{array}\right]^{\mathrm{T}}$ is joint angle variable array; $u$ is elastic deformation array; $\tau=\left[\begin{array}{llll}\tau_{M} & \tau_{\mathrm{m}} & 0\end{array}\right]^{\mathrm{T}}$ is input joint torque array of manipulators.

\section{The dynamic response of the flexible-macro rigid-micro manipulator}

In this paper, the above model is used to illustrate the effect on the dynamic characteristics separately from three aspects that structure parameters of the flexible-macro rigid-micro manipulator, physical parameters and motion parameters. In the following simulation example, flexible-macro manipulator and rigid-micro manipulator are equal cross-section rectangular beam. In the system narrate, $\mathrm{b}=0.016139 \mathrm{~m}, \mathrm{~h}=0.019518 \mathrm{~m}, \mathrm{E}=210 \mathrm{GPa}, \rho=7850 \mathrm{~kg} / \mathrm{m}^{3}, \mathrm{~L}_{1}=\mathrm{L}_{2}=1 \mathrm{~m}$, $\mathrm{L}_{3}=\mathrm{L}_{4}=0.2 \mathrm{~m}$, and $\quad \theta_{i}=\left\{\begin{array}{l}\theta_{\mathrm{o}}+A\left[\frac{2 t}{t_{\mathrm{o}}}-\sin \left(\frac{4 \pi t}{t_{\mathrm{o}}}\right) / 2 \pi\right], \mathrm{O} \leq t \leq \frac{t_{\mathrm{o}}}{2} \\ \theta_{\mathrm{o}}+A\left[2-\frac{2 t}{t_{\mathrm{o}}}+\sin \left(\frac{4 \pi t}{t_{\mathrm{o}}}\right) / 2 \pi\right], t>\frac{t_{\mathrm{o}}}{2}\end{array}\right.$

\subsection{The influence of structure parameters on the dynamic characteristics of the macro micro manipulator}

Change the length of the macro micro manipulator and then its influence on the dynamic characteristics can be seen from Fig. 3 to Fig. 4 . As $l=0.2 \mathrm{~m}$, the maximum deviation of $\mathrm{x}$ direction is $0.0426 \mathrm{~m}$ and the maximum deviation of y direction is $0.06 \mathrm{~m}$. As $l=0.4 \mathrm{~m}$, the maximum deviation of $\mathrm{x}$ direction is $0.1246 \mathrm{~m}$ and the maximum deviation of $\mathrm{y}$ direction is $0.159 \mathrm{~m}$. As $L=1 \mathrm{~m}$, the maximum deviation of $\mathrm{x}$ direction is $0.0426 \mathrm{~m}$ and the maximum deviation of $\mathrm{y}$ direction is $0.06 \mathrm{~m}$. As a result, the influence on the end error of flexible-macro rigid-micro manipulator increases along with the increase of manipulator length. The influence caused by length change of manipulator is greater on the end error of macro manipulators than on the one of micro manipulators.
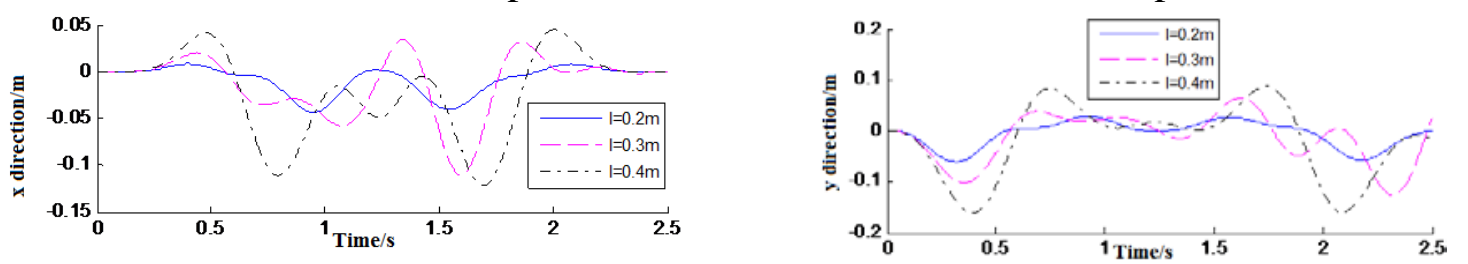

Fig.3. The $\mathrm{X}$ and $\mathrm{Y}$ direction end error of the manipulator of different length
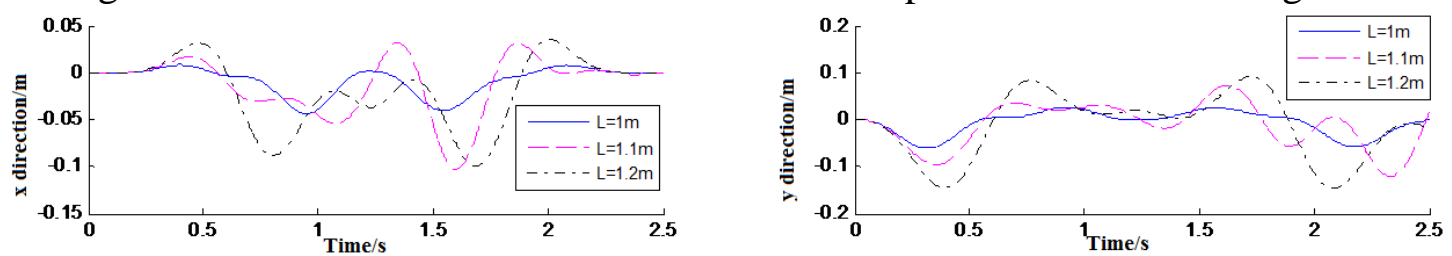

Fig.4. The $\mathrm{X}$ and $\mathrm{Y}$ direction end error of the manipulator of different length

As shown in Fig.5 and Fig.6, while $h=0.02 \mathrm{~m}$, the maximum deviation of $\mathrm{x}$ direction is $0.0313 \mathrm{~m}$ and the maximum deviation of $y$ direction is $0.0395 \mathrm{~m}$. While $\mathrm{h}=0.04 \mathrm{~m}$, the maximum deviation of $\mathrm{x}$ direction is $0.0064 \mathrm{~m}$ and the maximum deviation of $\mathrm{y}$ direction is $0.0086 \mathrm{~m}$. Thus it can be seen, the 
macro micro manipulator end error is very sensitive to manipulator section height. In order to reduce the end error of the macro micro manipulator, the section height of the manipulator can be increased appropriately.
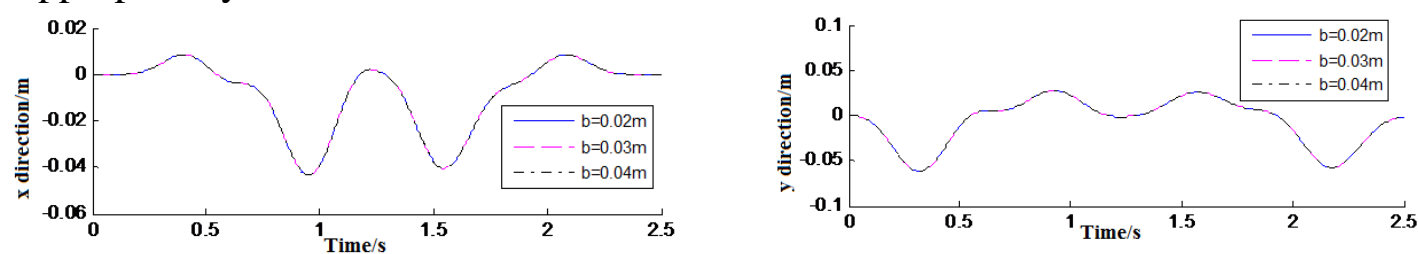

Fig.5. The $\mathrm{X}$ and $\mathrm{Y}$ direction end error of the manipulator of different section width
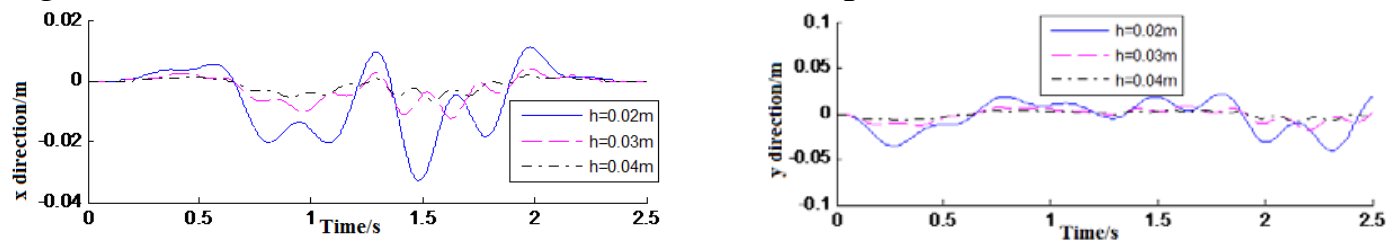

Fig.6. The $\mathrm{X}$ and $\mathrm{Y}$ direction end error of the manipulator of different section height

\subsection{The influence of physical parameters on the dynamic characteristics of the macro micro manipulator}

As is shown in Fig.7, while E=210GPa, the maximum deviation of $\mathrm{x}$ direction is $0.0426 \mathrm{~m}$ and the maximum deviation of $\mathrm{y}$ direction is $0.06 \mathrm{~m}$. While $\mathrm{E}=150 \mathrm{GPa}$, the maximum deviation of $\mathrm{x}$ direction is $0.0961 \mathrm{~m}$ and the maximum deviation of $\mathrm{y}$ direction is $0.1112 \mathrm{~m}$. So the appropriate selection of high elastic modulus materials can reduce the macro micro manipulator terminal trajectory error.

As is shown in Fig.8, while $\rho=7850 \mathrm{~kg} / \mathrm{m}^{3}$, the maximum deviation of $\mathrm{x}$ direction is $0.0426 \mathrm{~m}$ and the maximum deviation of y direction is $0.06 \mathrm{~m}$. While $\rho=5400 \mathrm{~kg} / \mathrm{m}^{3}$, the maximum deviation of $\mathrm{x}$ direction is $0.0378 \mathrm{~m}$ and the maximum deviation of $\mathrm{y}$ direction is $0.0412 \mathrm{~m}$. Therefore, the appropriate selection of low density materials can reduce the macro micro manipulator end trajectory error.
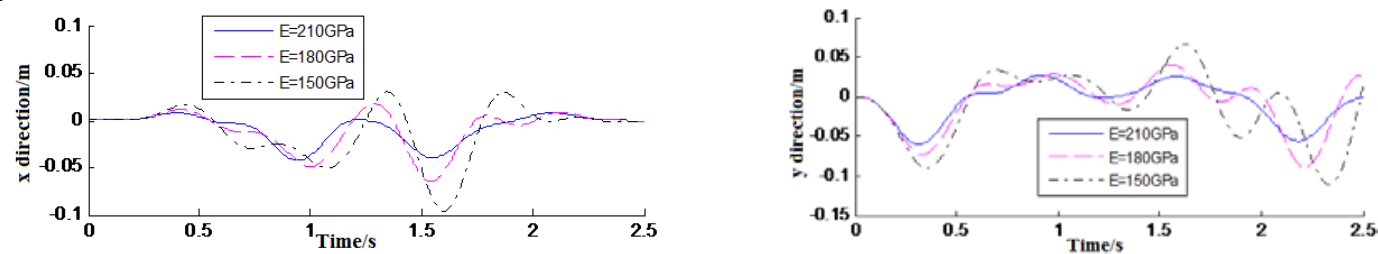

Fig.7. The $\mathrm{X}$ and $\mathrm{Y}$ direction end error of the manipulator of different elastic modulus
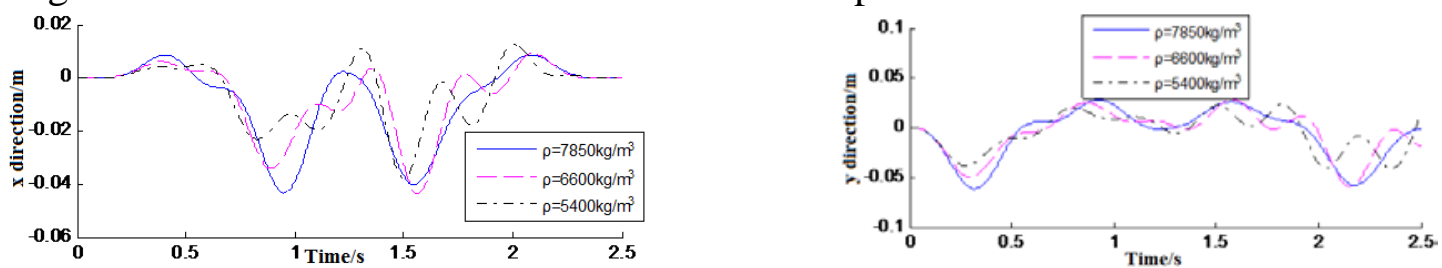

Fig. 8. The $\mathrm{X}$ and $\mathrm{Y}$ direction end error of the manipulator of different density

\subsection{The influence of motion parameters on the dynamic characteristics of the macro micro manipulator}

As is shown in Fig.9, while $\theta_{0}=0$, the maximum deviation of $\mathrm{x}$ direction is $0.0378 \mathrm{~m}$ and the maximum deviation of $\mathrm{y}$ direction is $0.0412 \mathrm{~m}$. While $\theta_{0}=\pi / 2$, the maximum deviation of $\mathrm{x}$ direction is reduced to $0.0051 \mathrm{~m}$ and the maximum deviation of y direction is reduced to $0.0016 \mathrm{~m}$. Therefore, increasing the initial phase angle appropriately can reduce the macro micro manipulator end trajectory error.

As is shown in Fig.10, while $A=\pi / 6$, the maximum deviation of $x$ direction is $0.0378 \mathrm{~m}$ and the 
maximum deviation of $y$ direction is $0.0412 \mathrm{~m}$. While $A=\pi / 2$, the maximum deviation of $x$ direction is increased to $0.0434 \mathrm{~m}$ and the maximum deviation of y direction is increased to $0.1136 \mathrm{~m}$. Therefore, decreasing the amplitude appropriately can reduce the macro micro manipulator end trajectory error.

As is shown in Fig.11, while $t_{0}=2 \mathrm{~s}$, the maximum deviation of $\mathrm{x}$ direction is $0.1044 \mathrm{~m}$ and the maximum deviation of y direction is $0.1365 \mathrm{~m}$. While $\mathrm{t}_{0}=3 \mathrm{~s}$, the maximum deviation of $\mathrm{x}$ direction is reduced to $0.0375 \mathrm{~m}$ and the maximum deviation of y direction is reduced to $0.046 \mathrm{~m}$. Therefore, increasing the cycle appropriately can reduce the macro micro manipulator end trajectory error.
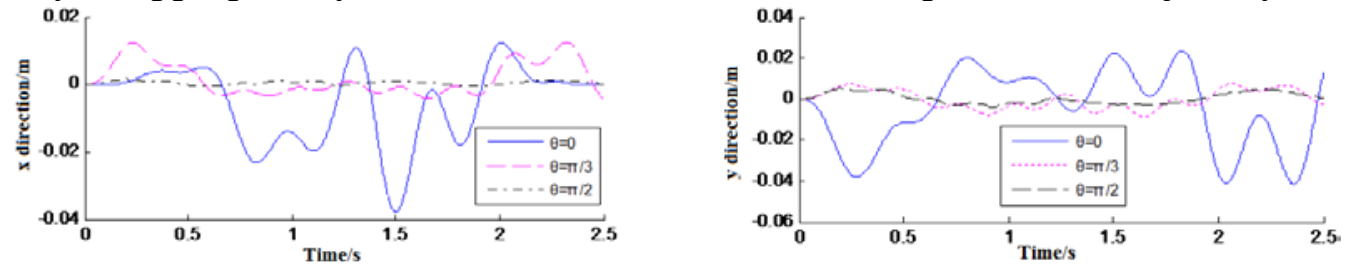

Fig.9. The $\mathrm{X}$ and $\mathrm{Y}$ direction end error of the manipulator of different initial phase angle
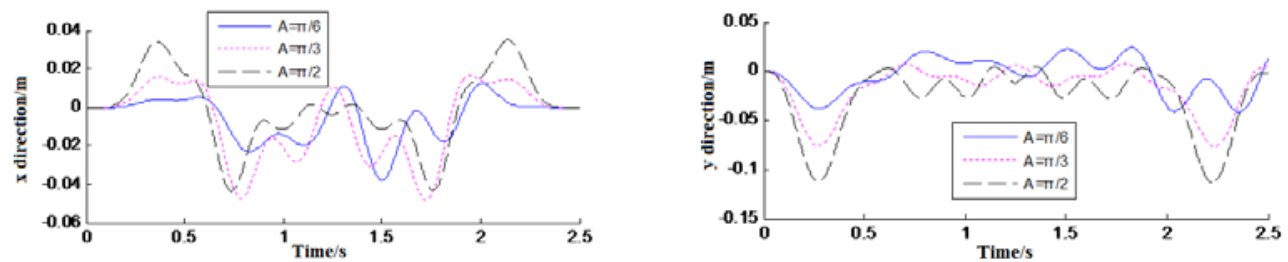

Fig.10. The $\mathrm{X}$ and $\mathrm{Y}$ direction end error of the manipulator of different amplitude
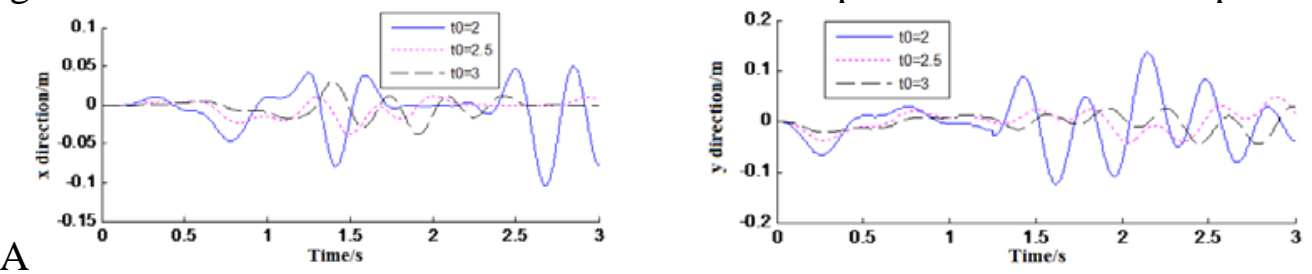

Fig.11. The $\mathrm{X}$ and $\mathrm{Y}$ direction end error of the manipulator of different cycle

\section{Conclusion}

The flexible-macro rigid-micro manipulator is a manipulator system with special structures. The relationship between dynamics model and its parameters is studied in this paper. The relationship between dynamics characteristics and its parameters is studied in this paper. Detailed simulation results have been given. In summary, many ways such as decreasing manipulator length, increasing section height, increasing elasticity modulus, increasing the density, increasing initial phase angle, decreasing amplitude or increasing cycle can all reduce the end error of the manipulator movement.

\section{References}

[1] Tokhi M O, Azad K M. Flexible Robot manipulators : Modelling, Simulation and Control [M]. IET, 2008.

[2] WANG Congqing ZHANG Chenglong.The dynamic control of free-floating flexible dual-arm space robot system [J].Journal of Mechanical Engineering, 200743 (10) 196-200.

[3] Wang Xucheng.The finite element method[M]. Tsinghua university press, 2003.

[4] Yim W, Singh S N. Nonlinear inverse and predictive end point trajectory control of flexible macro-micro Manipulators[C]. IFAC the 13th Triennial World Congress, 1996.97-102.

[5] Sharon A, Hogen N, Hardt D E. High bandwidth force and inertia reduction using a macro/micro manipulator system[C]. Proceedings of IEEE International Conference on Robotics and Automation, 1988.126-132. 